\title{
Validation of the QSCAT NRCS on the Advanced Neural Network NSCAT GMF and estimation of Neural Network QSCAT GMF
}

\author{
N. Tran, S. Thiria, M. Crepon \\ Laboratoire d'Oceanographie Dynamique et de Climatologie, \\ Universite de Paris 6, 4 place Jussieu, 75005 Paris, France \\ Tel: 33-1-44-27-27-09 Fax: 33-1-44-27-71-59 \\ E-mail: tran@lodyc.jussieu.fr, thiria@lodyc.jussieu.fr, mc@lodyc.jussieu.f \\ F. Badran \\ Centre d'Etude et De Recherche en Informatique du Conservatoire National \\ des Arts et Métiers, 292 rue Saint-Martin, 75003 Paris, France \\ Tel: 33-1-40-27-22-69 E-mail: badran@cnam.fr \\ M. Freilich \\ College of Oceanic and Atmospheric Sciences Oregon State University \\ 104 Ocean Admin Bldg Corvallis, OR 97331-5503, USA \\ Tel: (541) 737-2748 Fax: (541) 737-2064 E-mail: mhf@oce.orst.edu
}

\begin{abstract}
Neural networks are relevant statistical methods that we used to determine the GMFs of NSCAT and QSCAT scatterometer. They are well-suited for non-linear regression but they also can give more information than just the mean of a conditional distribution of data. We have also determined a function which estimates the conditional variance of QSCAT measurements following the previous works on NSCAT measurements.
\end{abstract}

\section{INTRODUCTION}

The wind vector on the ocean surface can be determined from measurements of radar backscatter (NRCS or $\sigma_{0}$ ) of the ocean by using a spaceborne scatterometer. The global coverage and frequent sampling give the spaceborne scatterometer advantages over other measurements. The SeaWinds scatterometer instrument of QuikSCAT mission (NASA) was launched in June 19, 1999 to fill the gap created by the loss of data from NASA Scatterometer (NSCAT), when the ADEOS-1 satellite lost power in June 1997. It is also a Ku-band radar. It has antenna beams at two elevation angles giving a conical scan (concentric circles on the surface) and acquires measurements in vertical (VV-pol) and horizontal (HH-pol) polarizations of NRCS. This conical design represents a change from the fixed fan-beam antennas of previous spaceborne scatterometers (NSCAT and ERS-1/2).

Analysis and modeling of polarized (VV-pol and HH-pol) ocean backscatter data is an active research topic for wind scatterometry application. And to date only empirical models are used to derive the wind. These empirical relationships, known as Geophysical Model Functions (GMF), relate statistically the backscatter measurements to the geophysical parameters, are derived from collocated observations with European Centre for Medium-Range Weather Forecasts (ECMWF) model winds. GMF are forward model, i.e., the backscatter is expressed as a function of wind speed, wind direction and incidence angle. To retrieve a wind from measurements of $\sigma_{0}$, the model must be inverted. If the values of the backscatter do not change, the empirical function forms can be quite different because the backscatter depends very nonlinearly on wind speed and directions. Moreover other geophysical parameters may also affect $\sigma_{0}$ such as the wave height, the wave direction, the sea surface temperature and the rain since they modify the sea surface response to the wind action. These parameters, which are thought to act at second order, are not be taken into account in the determination of the geophysical models.

Errors in the final retrieved wind vectors are then dependent not only on the model function, but also on errors in the backscatter measurements. There are two different sources of error in the $\sigma_{0}$ which had to be considered: (1) the error in the determination of the attitude of the spaceborne and error inherent in the radar receiver and mesurement strategy (communication noise) and (2) the variability in the observed $\sigma_{0}$ due to the effects of unmodeled geophysical parameters on $\sigma_{0}$ (modeling error). And the maximum likelihood wind retrieval algorithm usually used require knowledge (or at least a good estimate) of the variance of the $\sigma_{0}$ measurements and thus of these two error terms. While the first error can be analytically computed based on the instrument design parameters, suitable modeling 


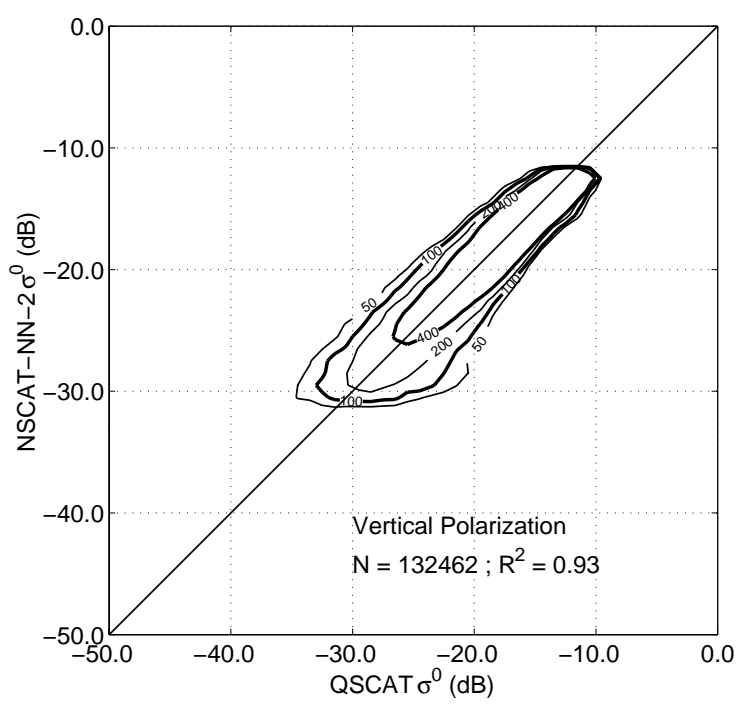

Figure 1: Scatter diagrams of the estimations of QSCAT measurements by NSCAT-NN-2 neural model versus the QSCAT measurements for the vertical polarization.

error estimates for existing model functions are not presently available.

The challenges of scatterometry thus include development of robust Geophysical Model Function and accurate characterizations of measurement error statistics. This is done in the present paper by using multilayer perceptrons, a particular class of neural networks.

\section{NON-LINEAR REGRESSION BY NEURAL NETWORKS}

Our approach is to determine an empirical function which models $\sigma_{0}$ in each polarization with respect to the incidence angle $\theta$, the wind speed $\mathrm{U}$ and the azimuth direction $\chi$, which represents the wind direction relative to the radar beam direction. Due to the significant nonlinearity in the function, we choose an empirical model able to deal with nonlinear regression. In the following, we use a Neural Network (NN) methodology which has been used with success for modeling complex functions $[1,2]$. NN provides empirical statistical nonlinear models estimated from observations in the form of a continuous function [3] which in this case is expressed by a functional equation of the form :

$$
\sigma_{0}=F(\theta, U, \chi) .
$$

We use a particular architecture for the neural network model, the so-called Multi-Layer Perceptrons (MLP) which are the most commonly used for nonlinear regression.

We assume that each observation $\sigma_{0}{ }^{i}$ is the sum of the true signal $\sigma_{0 *}{ }^{i}$ and a Gaussian noise $e^{i}$ with zero mean, and variance $\operatorname{var}\left(e^{i}\right)$ :

$$
\sigma_{0}{ }^{i}=\sigma_{0 *}{ }^{i}+e^{i} .
$$

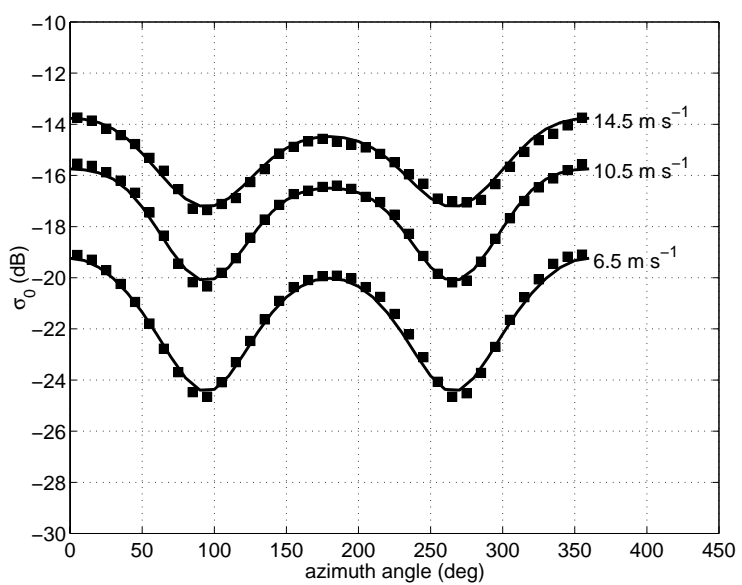

Figure 2: Azimuthal modulations of QSCAT measurements estimated for three wind speed for the vertical polarization. The square show the bin-averaged mean of $\sigma_{0}$. The full line shows the QSCAT-NN model.

Thus as stated in neural networks theory, $F(\theta, U, \chi)$ gives an estimate of the conditional mean of the measurements:

$$
F(\theta, U, \chi) \approx E\left[\sigma_{0} / \theta, U, \chi\right]
$$

The knowledge of $\operatorname{var}\left(e^{i}\right)$ gives useful information on the response of the scatterometer and the accuracy of the GMF. We assume that the variance of the observed $\sigma_{0}$ is a function of the true $\sigma_{0 *}$, the wind speed $\mathrm{U}$, the azimuth direction $\chi$ and the incidence angle $\theta$. To compute this variable, we use a maximum likelihood estimation. This approach is also based on neural networks and described in [4].

\section{RESULTS}

We only present a few results for the vertical polarization in the following. Results for the horizontal polarization are not shown. Fig. 4 shows the scatter diagrams of the $\sigma_{0}$ estimated respectively by a previous Ku-band neural GMF which has been calibrated with NSCAT measurements (NSCAT-NN2) and by a new neural GMF estimated on QSCAT measurements themselves (QSCAT-NN) versus the observed QSCAT $\sigma_{0}$. The data are almost along the perfect line and the correlation coefficients $R^{2}$ are of 0.93 for the two models. NSCATNN-2 is a good model for QSCAT scatterometer but we can note that the estimations of the very low values of QSCAT $\sigma_{0}$ are better when the model is calibrated on the same data.

The estimation of a variance function is more difficult than the estimation of the conditional mean. The preliminary result shown for QSCAT measurements in the Fig. 3 for a wind speed of $12.5 \mathrm{~m} \mathrm{~s}^{-1}$ and the vertical polarization is quite realistic. The data are almost into the error bars at two standard deviations. 


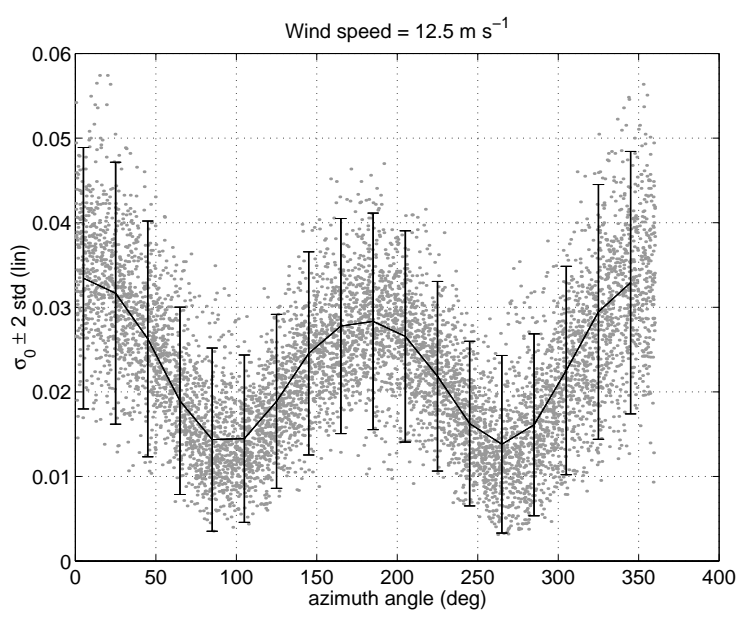

Figure 3: Distribution of QSCAT measurements for wind speed ranges of $12.5 \pm 0.5 \mathrm{~m} \mathrm{~s}^{-1}$ with respect to the azimuth angle and neural estimations of the mean and bar corresponding to 2 standard deviations for a wind speed of $12.5 \mathrm{~m} \mathrm{~s}^{-1}$ for some azimuth angles.

\section{CONCLUSION}

The NSCAT and QSCAT neural network GMFs were derived by using backscatter measurement respectively and ECMWF analysis winds as input and approximate well this relationship.

But unfortunately, all observation systems contain error. This means that when we assume that one measurement (wind vector) represents the true state and calibrate the other $\left(\sigma_{0}\right)$ against it, pseudobiases may occur $[5,6]$. By using triple collocations, $[5,6]$ propose a method to provide an error characterization for all three noisy observation systems of wind (buoys, analysis from meteorological model and scatterometer winds) which should lead to reduce biased estimates of regression coefficients.

The more general problem is then to determine the regression function when dealing with both noisy wind vectors and backscatter measurements and required the use of a more sophisticated neural model proposed by [7].

The use of this sophisticated neural model with triple collocations of wind measurements should lead to an improvement of our knowledge on the relationship between the "true" ocean wind fields and the radar backscatter of the ocean.

\section{REFERENCES}

[1] Thiria, S. and Badran, F. and Mejia, C. and Crepon, M., "A neural network approach for modelling nonlinear transfer functions: Application for wind retrieval from spaceborne scatterometer data", J. of Geophys. Res., 98, C12, 22,827-22,841, 1993

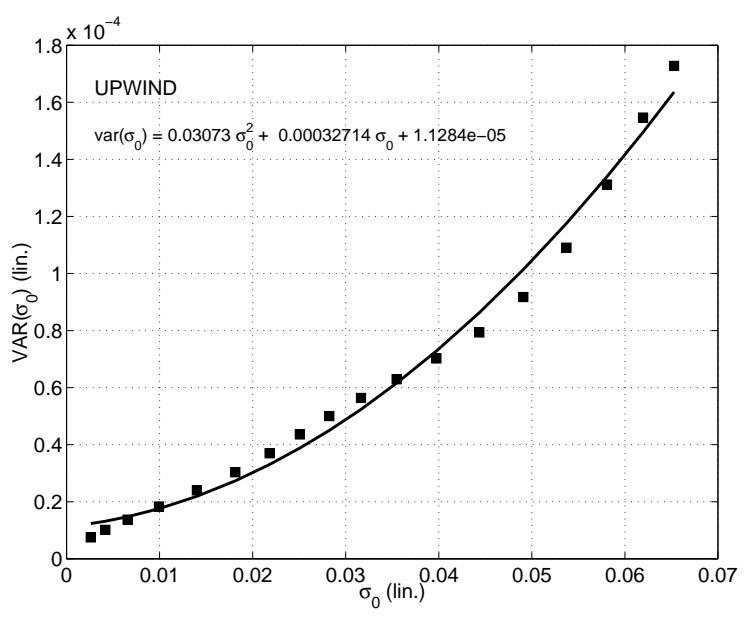

Figure 4: The estimated variance by the neural model with respect to the estimated mean of $\sigma_{0}$ by QSCAT-NN model for the vertical polarization and the upwind direction. This relationship has a quadratic form.

[2] Mejia, C. and Thiria, S. and Badran, F. and Tran, N. and Crepon, M.,"Determination of the geophysical model function of ERS-1 scatterometer by the use of neural networks", 103, C6, 12,853-12,868, 1998

[3] Bishop, C. M., Neural networks for pattern recognition, Oxford University Press, 1995

[4] Mejia, C. and Badran, F. and Bentamy, A. and Crepon, M. and Thiria, S. and Tran, N., "Determination of the geophysical model function of NSCAT scatterometer and its corresponding variance by the use of neural networks", J. of Geophys. Res., 104, C5, 11,539-11,556, 1999

[5] Freilich, M. H. and Dunbar, R. S., "The accuracy of the NSCAT-1 vector winds: Comparisons with National Data Buoy Center buoys", J. of Geophys. Res., 104, C5, 11,231-11,246, 1999

[6] Stoffelen, A., "Error modeling and calibration: towards the true surface wind speed", J. of Geophys. Res., 103, C4, 7755-7766, 1998

[7] Badran, F., Stephan, Y., Metoui, N. and Thiria, S.,”A general formulation of nonlinear least squared regression using multi-layered perceptrons", in preparation, 2000 